\title{
The Impacts of Collaboration and Cordination of Architectural and Engineering Projects Developed with BIM in Reducing Design Interferences
}

\author{
Dilson Albuquerque Pessoa Fonseca, Max Lira Veras Xavier de Andrade \\ UFPE, Brasil \\ dilsonfonseca97@gmail.com \\ max.andrade@ufpe.br
}

\begin{abstract}
This paper addresses the importance and development of cultural transformations involving the design process in architecture and the advent of Building Information Modeling (BIM) in civil construction activities and how its implementation in a coordinated, collaborative and interoperable way contributes to a diagnosis of Clash Detection between diferentes design projects, before building construction, saving excessive costs and rework. Taking as its main reference the BIM Maturity Matrix of Succar (2009), the proposed BIM Project Integration Maturity Matrix contributes to the awareness of bringing designers and builders closer to design activities, to encourage the integration of design processes involving the building, to consolidate an environment of ease of communication between participants, the organization of documentation and, above all, prioritize the compatibility between projects to avoid conflicts, excess costs and rework, resulting in a higher quality of the final project.
\end{abstract}

Keywords: Coordination; Building Information Modeling (BIM); Design interferences; Integrated Project; Design process with BIM.

\section{Introdução}

O estudo da detecção de interferências (Clash Detection) de projeto é um tema que vem gerando importantes discussões na indústria da construção civil, principalmente com a consolidação de práticas projetuais baseadas no Building Information Modeling (BIM ou Modelagem da Informação da Construção). O desenvolvimento de projetos em BIM, quando estruturados em práticas colaborativas e integradas (Sacks et al. 2018) apoiadas no projeto simultâneo (Fabrício 2002), em modelos de comunicação e na interoperabilidade podem 
gerar transformações na prática de projeto que implique na redução das interferências entre disciplinas, com subsequente melhoria na qualidade da solução a ser construída no canteiro de obras.

É importante lembrar que até meados dos anos 60 os projetos eram desenvolvidos e construídos dentro de uma mesma empresa, o que permitia trabalhos mais colaborativos e uma visão mais integrada entre as diversas especialidades (Coelho; Novaes, 2008). A terceirização das atividades de projeto e o seu distanciamento com a atividade de construção do edifício, principalmente a partir do final dos anos 70 e 80 no Brasil, além do aumento da complexidade dos edifícios nessa época e em períodos subsequentes, resultaram em uma série de problemas de compatibilidade e coordenação, gerando altos índices de desperdício, com um número crescente no nível de interferências entre as diferentes disciplinas. Muitas dessas interferências passam a ser resolvidas no canteiro de obras, gerando retrabalhos e entulhos de construção.

Como uma forma de reduzir esses impactos no custo da construção e no meio ambiente, a indústria da construção passou a valorizar, principalmente a partir dos anos 90, as atividades de coordenação e compatibilização, associadas a políticas de gestão do projeto e da obra (Ruschel et al. 2013).

Todavia, mesmo com todas essas tentativas, as atividades de colaboração ainda aconteciam de maneira isolada, resultado de ações paralelas de especialistas. Para Ruschel et al. (2013), a atividade de projeto tradicional ainda ocorre de maneira discreta, individual e paralela. Para esses mesmos autores, as atividades de projeto comportam-se como atividades desenvolvidas por especialistas, que os fazem segundo os seus conhecimentos individuais, abordando as questões de projeto sob perspectivas estritamente ligadas às suas profissões, o que resulta em uma colaboração altamente segmentada.

A consolidação das práticas de projeto baseadas no BIM, em conjunto com práticas colaborativas e coordenadas pode, segundo Leusin (2018), gerar uma redução de até $90 \%$ nos processos de revisões de projeto, ou seja, nos retrabalhos. Isso pode também trazer impactos significativos nos desperdícios do canteiro de obras, reduzindo também o custo da construção. O BIM, ao estar vinculado a práticas colaborativas, integradas e a tecnologias de comunicação/colaboração da informação, traz um impacto significativo na detecção das incompatibilidades entre projetos, que até recentemente eram apenas identificadas durante a obra. Contudo, Leusin (2018) ressalta que o não entendimento de que a implementação do BIM precisa estar associada a uma mudança na cultura de projeto, pode gerar casos de insucesso na implantação de processos BIM na organização. Dessa forma, é necessário ressaltar a compreensão de que a implantação do BIM precisa estar vinculada 
à novas práticas de concepção e desenvolvimento de projetos que sejam essencialmente colaborativas e integradas, apoiadas em uma base tecnológica que dê suporte à sistemas de comunicação, tecnologias de bancos de dados, servidores, softwares e hardwares.

É dentro desse contexto que o presente trabalho a ser apresentado nesse artigo se fundamenta. O mesmo tem como objetivo desenvolver e apresentar uma Matriz de Maturidade de Integração de Projeto BIM, tendo como base o nível de Detecção de Interferências entre o projeto de arquitetura e seus complementares. O que se espera com essa matriz é identificar a qualidade da coordenação e colaboração dos projetos, tendo como base, o nível de interferência entre as diferentes disciplinas envolvidas. Espera-se com isso, mostrar que projetos mais colaborativos e coordenados resultam em um menor nível de interferências (+Clash Avoidance) e projetos menos colaborativos e integrados resultam em mais interferências entre projetos (+Clash Detection).

\section{Metodologia}

Para alcançar os objetivos estabelecidos, os procedimentos da pesquisa foram divididos em três momentos. O primeiro procedimento consistiu na revisão da literatura sobre os processos de projetos tradicionais de arquitetura, sobre o projeto simultâneo, BIM, práticas colaborativas e integradas apoiadas no BIM. Também foram analisados os Usos BIM propostos por Succar et al. (2016) e, especificamente o uso de Detecção de Interferência (Clash Detection) e Evasão de Choque (Clash Avoidance) trazido por Akponeware e Adamu (2017). Por fim, foi estudando a Matriz de Maturidade de Succar (2009) e utilizando seus fundamentos como base para a proposição da matriz de maturidade a ser apresentada neste trabalho.

Após a revisão de literatura na área da pesquisa, partiu-se para a segunda etapa da pesquisa. Essa consistiu na realização de estudos de casos em três escritórios de projeto que já vêm trabalhando com o BIM há vários anos na cidade do Recife (Pernambuco/BR). A ideia era compreender melhor as transformações nas práticas de projeto com o BIM, por meio da avaliação do nível de Detecção de Interferências entre o projeto de arquitetura e complementares. O que se esperava identificar era se, para aqueles escritórios com práticas mais colaborativas e integradas a quantidade de detecção de interferência (Clash Detection) era maior ou menor do que aqueles escritórios, que por mais que tivessem uma prática bem consolidada no uso de ferramentas BIM, não tivessem práticas de colaboração e integração bem consolidadas. Será que neste último caso, resultaria numa maior quantidade 
de detecção de interferência (Clash Detection) entre os projetos e uma menor evasão de choque? Será que isso poderia ser identificado em uma matriz de maturidade?

Os estudos de casos se deram pela avaliação do perfil dos escritórios de projeto pesquisados e por entrevistas com os arquitetos coordenadores desses escritórios. Por meio da entrevista foi possível compreender melhor o processo de projeto desses escritórios, os benefícios e as limitações ocorridos nos mesmos com a adoção do BIM. Também foi analisado o nível de colaboração entre a disciplina de arquitetura e as demais disciplinas em cada um desses três escritórios. Por fim, foi analisado o nível de detecção de interferência em três projetos escolhidos, um projeto de cada escritório pesquisado. Outras informações também foram adquiridas com as entrevistas: os processos de comunicação e colaboração entre os membros envolvidos no projeto; como acontecem as trocas de arquivos entre as diferentes disciplinas; onde as informações são armazenadas; há uso de Ambiente Comum de Dados (CDE) ou servidor BIM nos escritórios, os projetistas usam esses ambientes? Como acontecem os relatórios de interferências e como as interferências são comunicadas para os diferentes projetistas. Tudo isso foi realizado visando entender melhor os processos de colaboração e comunicação em cada um desses escritórios pesquisados.

A partir das investigações em processo de projeto de arquitetura e com base no destrinchamento e entendimento da Matriz de Maturidade proposta por Succar (2009) alinhada aos estudos de casos realizados em escritórios de projeto foi possível identificar uma relação direta entre o nível de colaboração e coordenação de projetos em BIM e a quantidade de interferência de projetos, detectadas por cada um desses escritórios. Isso se deu na última etapa do trabalho a ser discutida a seguir.

\section{Resultados}

A Matriz de Maturidade concebida por Succar (2009) foi construída a partir do contexto de que o BIM é formado por um conjunto de políticas, processos e tecnologias. Dado os resultados encontrados nos estudos de caso, é possível observar que o desempenho do BIM possui uma relação direta com a aplicação dessas políticas, processos e tecnologias de adoção do BIM empregadas nas empresas. Com os cenários analisados em cada uma das três empresas pode-se compreender que esses três conjuntos cumprem um papel de interdependência. Ou seja, para alcançar o maior grau de benefícios na adoção do BIM é importante que os projetistas não se limitem a atender apenas um desses campos de atuação do BIM. 
A investigação se deu em três empresas de projeto denominadas aqui de: Empresa A, Empresa B e Empresa C. Os resultados da investigação mostram, ainda que preliminarmente, que mesmo que uma empresa tenha um nível de adoção BIM ainda inicial, acredita-se que os benefícios do BIM vão aparecer de modo mais assertivo apenas àquelas empresas que possuam sempre um de maturidade proporcional entre políticas, processos e tecnologias. O fato de uma empresa ter um bom avanço do uso de tecnologias BIM, mas pouco avanços em processos e políticas fazem dificultar o avanço na adoção do BIM. Esse foi o caso da Empresa B estudada, que embora já usasse algumas tecnologias e ferramentas BIM faz muitos anos a falta de processos e políticas bem definidos dificultaram o avanço da maturidade BIM desta empresa. Já a empresa $A$, mesmo com um conhecimento tecnológico não tão sofisticado, comparado à empresa $B$, apresentou um nível de maturidade equivalente entre as políticas, processos e tecnologias. Para o caso dessa empresa constatou-se que a mesma apresentava um maior nível de maturidade na adoção do BIM, com impactos, inclusive na redução do nível de interferência entre as várias disciplinas de projeto.

Essa interdependência entre os conjuntos, mencionada acima também é evidenciada nas instruções de uso que são elucidadas na matriz de maturidade do Succar (2009): "Evite calcular pontuações totais (por linhas ou colunas), pois esses totais podem ser enganosos. " (BIM Initiative, 2017, p. 2). Essa recomendação implica que a pontuação de uma área-chave de maturidade não pode ser levada em consideração isoladamente, mas apenas na conjuntura integrada entre estas, ou seja, o resultado final. Isso ilustra as relações de continência entre os campos de atuação do BIM, capacidades BIM e o grau de maturidade encontrado.

É perceptível como esses resultados mostram a necessidade da adoção do BIM em processo de concepção e desenvolvimento de projeto que evidenciem a coordenação e colaboração de modo a reduzir as incompatibilidades entre os projetos. Isso é evidenciado a partir do estudo de caso na Empresa A. Esta empresa estruturou o processo de implementação do BIM em práticas integradas desde o momento inicial do processo projetual, alinhados à adoção de tecnologias e práticas apoiadas em um BEP bem estruturado. Isso permitiu reduzir a quantidade de interferência entre os projetos. Ou seja, + clash avoidance e - clash detection.

\subsection{Matriz de Maturidade de Integração de Projeto BIM}

A partir da investigação dos fatores que corroboram a eficácia do método avaliativo proposto por Succar (2009) com a Matriz de Maturidade BIM, foi possível estabelecer os condicionantes que definiram a estrutura e o 
direcionamento a ser trazido pela Matriz de Maturidade de Integração de Projetos BIM.

Como já mencionado anteriormente, Succar (2009) define o BIM como um conjunto de processos, políticas e tecnologias. Essa definição permeia toda lógica de construção do seu método de avaliação. Percebe-se que dentro do processo de projeto em BIM, esse conjunto também é definido por outros três aspectos que também possuem uma relação de interdependência e continência para garantir o melhor aproveitamento dos benefícios trazidos pelo BIM, de acordo com a Figura 01.

\section{Processos \\ Políticas}

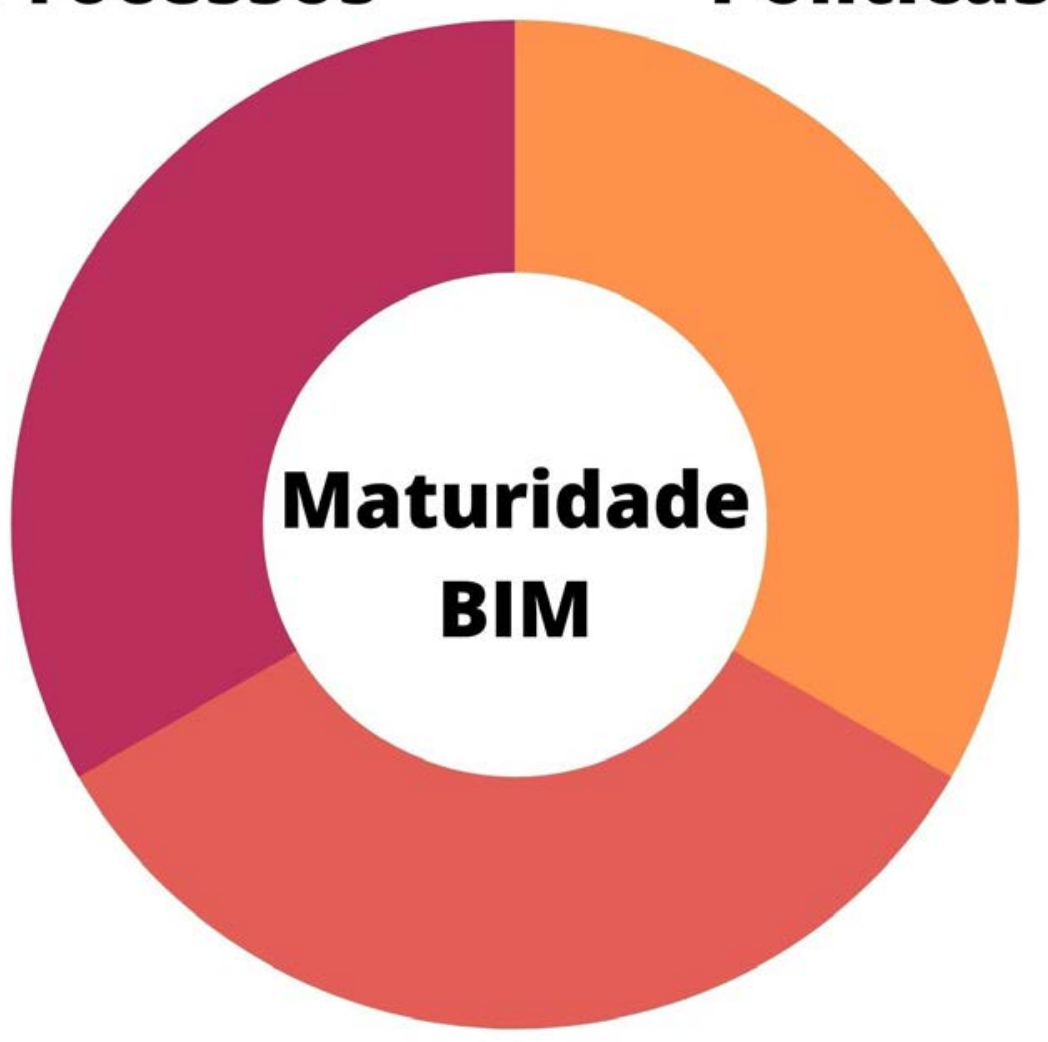

\section{Tecnologias}


construtivas, quanto de compromisso com prazos de entrega. Também é importante notar que essa alta incidência de conflitos pode advir de uma baixa interoperabilidade, resultando em conflitos geométricos entre os elementos, por falta de parâmetros informativos de saída dos arquivos trocados.

Definido: Neste índice, geralmente, já há um planejamento quanto às mudanças em relação aos processos de trabalho e já existe a figura do coordenador BIM supervisionando as etapas e buscando o desenvolvimento da integração dos projetistas, porém, em estágio inicial. Esse índice é caracterizado por uma razoável quantidade de conflitos projetuais, porém, já existe uma enorme vantagem em relação ao processo de projeto tradicional, no qual vários desses conflitos apenas seriam denotados na obra.

Integrado: Neste nível, existe uma grande coordenação e compatibilização entre os projetistas desde o momento da concepção do projeto, geralmente monitorada e faseada pelo Coordenador BIM, o acesso aos modelos do projeto é feito em tempo real e da forma mais atualizada possível e com uma padronização de saída dos arquivos para os projetistas participantes, buscando sempre otimizar as informações trocadas. É caracterizado por uma inexistência ou baixa incidência de interferências, tendendo ao mínimo, pois a integralização dos projetos permite sempre um alto grau de compatibilidade entre si.

Assim como a matriz referenciada, a Matriz de Maturidade de Integração de Projeto BIM também possui suas instruções de uso, são elas:

- Identificar a pessoa mais apta a conduzir a avaliação, dado que o resultado advém do conhecimento dos processos de trabalho, conhecimento das ferramentas BIM, cultura organizacional e a gestão de coordenação de projetos;

- A avaliação deve ser feita em grupo, preferencialmente com profissionais que cumprem diferentes papéis dentro da organização e processo de projeto;

- Usar as pontuações recomendadas (10-40) ou cores para iluminar o que foi conseguido até aquela data. Por exemplo, use a Cor $A$ se a integração descrita dentro da célula não foi atingida até a data. A Cor B se a integração foi parcialmente atingida; e a Cor $\mathrm{C}$ se a integração descrita foi totalmente atingida; 
- Para obter o resultado final, é necessário utilizar uma média aritmética, ou seja, dividir a pontuação encontrada pelo número de áreas-chaves que compõem a matriz;

- É considerável que os resultados encontrados sejam discutidos, buscando identificar melhorias a serem implantadas nos processos. É importante visar sempre uma melhoria global do que em uma única área, visto que os processos tendem sempre a serem integrados.

Tabela 01. Matriz de Maturidade de Integração de Projeto BIM.

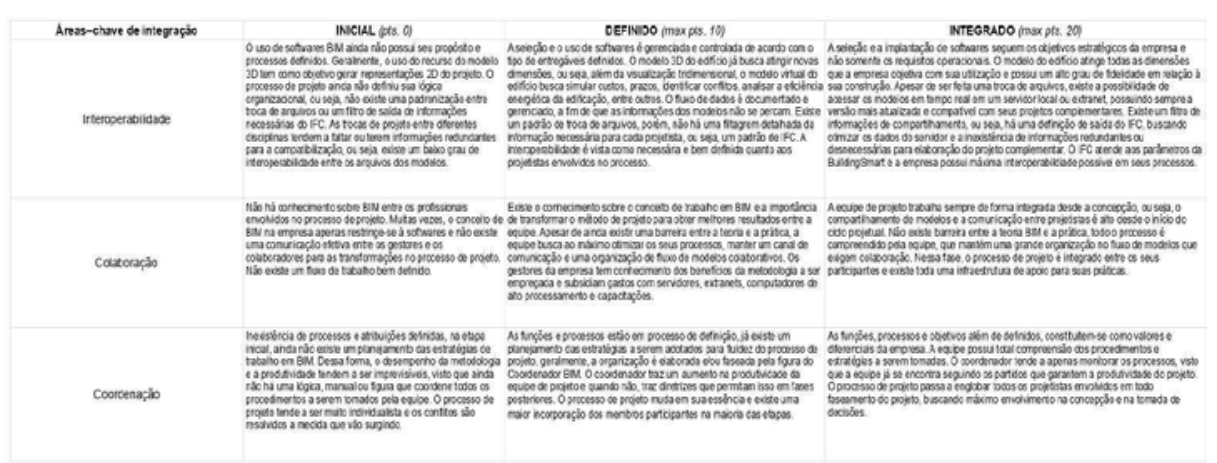

Fonte: Elaborado pelo autor, 2021. 


\section{Considerações Finais}

Diante de novas demandas estimuladas pelo setor da AECO, o arquiteto e urbanista encontra-se em um ambiente de transformação. Para atingir novas metas que os setores vêm solicitando, faz-se necessário investigar os processos que permeiam o fazer arquitetônico e sua operação. Dessa forma, a presente pesquisa buscou identificar metodologias, ferramentas ou conceitos que venham a auxiliar a produtividade do arquiteto e urbanista.

O presente trabalho buscou fazer um paralelo entre as transformações que englobam o processo de projeto, utilizando o BIM como conceito base e a contribuição dos métodos de avaliação para a análise e o desenvolvimento de novas estratégias de trabalho. Utilizando escritórios de projeto para ilustrar os estudos de caso, foi possível estabelecer relações entre os processos de concepção e desenvolvimento de projetos que fossem mais ou menos colaborativos e integrados com a ocorrência de interferências entre projetos de arquitetura e complementares. O texto também mostra como a Matriz de Maturidade BIM proposta por Succar (2009) firmou-se como um dos principais métodos de avaliação para mensurar a implementação do BIM em empresas. Essa discussão também acarretou na proposição de um novo método avaliativo, porém, com uma outra vertente, buscando compreender que fatores contribuem para a fluidez do projeto arquitetônico a partir da premissa que o setor da AECO, atualmente, busca por economia de custos. Nesse sentido, a Matriz de Maturidade de Integração de Projeto BIM busca a conscientização que a interoperabilidade, a colaboração e a coordenação de projetos dentro das práticas de concepção e desenvolvimento de projeto são práticas essenciais para a otimização dos fluxos de trabalho e com menor incidência de conflitos entre as diversas disciplinas.

A utilidade da Matriz de Maturidade de Integração de Projeto BIM nas empresas estudadas permitiu compreender como a colaboração, coordenação e interoperabilidade são essenciais para a concepção e desenvolvimento de projetos mais integrados, e, portanto, com menos conflitos entre as diferentes disciplinas. 


\section{Referências}

Akponeware, A.; Adamu, Z. (2017). Clash Detection or Clash Avoidance? An Investigation into Coordination Problems in 3D BIM. MDPI.

BIM Initiative (2017), 211in. Model Uses Table, v1.26. Acesso em: 10 de Junho, 2021. Disponível em: https://bimexcellence.org/wp-content/uploads/211in-Model-UsesTable.pdf.

BIM Initiative (2017), 301in. BIM Maturity Matrix, v1.22. Acesso em: 10 de Junho, 2021. Acesso em: 10 de Junho, 2021. Disponível em: https://bimexcellence.org/wpcontent/uploads/301in.PT-Matriz-de-Maturidade-BIM.pdf.

Fabrício, M. (2002). Projeto Simultâneo na construção de edifícios. Tese (Doutorado em Engenharia Civil) - USP

Leusin, S. (2018). Gerenciamento e Coordenação de Projetos BIM: Um guia de ferramentas e boas práticas para o sucesso de empreendimentos. 1. ed. Rio de Janeiro: Elsevier, 2018. ISBN 9788535290042.

Ruschel, R. ; Valente, C. ; Cacere, C. ; Queiroz, E. (2013). O papel das ferramentas BIM de integração e compartilhamento de no processo de projeto na indústria da construção civil. REEC - Revista Eletrônica de Engenharia Civil, Vol. 7.

Succar, B. (2009). Building information modelling framework: a research and delivery foundation for industry stakeholders. Automation in Construction, 18(3), 357-375.

Succar, B. (2009). Building Information Modeling maturity matrix. In J. Underwood \& U. Isikdag (Eds.), Handbook of research on Building Information Modelling and construction informatics: concepts and technologies (pp. 65-103): Information Science Reference, IGI Publishing.

Succar, B.; Sher, W. ; Williams, A. (2009). Measuring BIM performance: Five metrics. In: Architectural Engineering and Design Management. School of Architecture and Built Environment, University of Newcastle, Australia.

Succar, B; Saleeb, N. ; Sher, W. (2016). Model Uses: Foundations for a Modular Requirements Clarification Language, Australasian Universities Building Education. (AUBEA2016), Cairns, Australia. 